\title{
Reproducing Proximal Femur Anatomy with Custom Stems
}

\author{
Elhadi Sariali, Alexandre Mouttet, Xavier Flecher, \\ and Jean Noel Argenson
}

\subsection{Introduction}

The proximal femur anatomy is highly variable between hip osteoarthritic patients [1-4]. This variability may render reliable restoration of the native hip anatomy and biomechanics difficult when performing total hip arthroplasty (THA) with conventional off-the-shelf stemmed femoral components. Poor restoration of biomechanical hip parameters such as femoral offset (FO), leg length (LL), and the femoral anteversion (FA) may compromise clinical outcome due to the resultant limp [5], edge loading [6], prosthetic impingement, and dislocation [5]. For instance, as little as a $15 \%$ decrease in FO reduces the abductor moment arm and hampers gait [7], suggesting that accurately restoring the FO is important, especially for younger patients with high functional demands.

\section{E. Sariali $(\bowtie)$}

Hôpitaux Universitaires La Pitié Salpêtrière-Charles Foix, AP-HP, Paris, France

A. Mouttet

Polyclinique Médipôle Saint-Roch,

Perpignan, France

X. Flecher · J. N. Argenson

Department of Orthopaedics and Traumatology, Aix Marseille Univ, APHM, CNRS, ISM, Sainte-

Marguerite Hospital, Institute for Locomotion, Marseille, France

e-mail: Jean-noel.ARGENSON@ap-hm.fr
To assist surgeons in reproducing proximal femur anatomy in THA, conventional stems are typically available in two neck-shaft angles and two femoral offsets. Nevertheless, restoration of patient-specific femoral anteversion remains technically challenging-particularly for uncemented stems. Femoral stems with modular necks have therefore been developed to assist in the restoration of hip biomechanics (FO, LL, and FA) and to reduce the risk of prosthetic impingement. However, this results in excessive corrosion at the modular junction and leads to unacceptable rates of prosthetic neck fracture and adverse local tissue reaction to metal debris [8]; this has stymied their widespread adoption. The use of proximally loaded (metaphyseal fixation) custom stems has been proposed to precisely restore patient-specific proximal femur biomechanical parameters [9]. Their long-term clinical outcomes are excellent, with a survival rate of $97 \%$ at 20-year follow-up, including in very active below 50-year-old patients [10].

However, custom stems require threedimensional (3D) imaging and planning, a longer lead time before surgery to allow for manufacture, and are typically more expensive than conventional stems. Therefore, it remained unclear what proportion of THA patients requires a custom stem to achieve an accurate $3 \mathrm{D}$ restoration of proximal femur anatomy. To address this question, we conducted a prospective observational study between January 2009 and November 
2014, including all patients who underwent a 3D-planned primary THA using either an anatomic proximally hydroxyapatite (HA)-coated cementless modular-neck stem (off the shelf SPS ${ }^{\circledR}$ stem, Symbios, Switzerland) or a custom stem (Symbios, Switzerland).

\subsection{Methods}

Cohort description. Between 2009 and 2014, 578 consecutive patients underwent 3D-planning guided THA through a minimal invasive direct anterior approach. They were composed of 284 women and 294 men, aged 61 years $( \pm$ SD 13$)$ with a mean BMI of $26.5 \pm 5$. To restore hip biomechanics using $3 \mathrm{D}$ reconstruction, our prespecified guidelines determined that a custom stem was required in $72(12 \%)$ patients composed of 40 women and 32 men aged 48 years (SD 15.4) with a mean BMI of $26.7 \pm 5 \mathrm{~kg} / \mathrm{m}^{2}$, amongst whom 12 patients had previous hip surgery. In the custom group, the most frequent etiologies were DDH in 33 (46\%) patients, primary osteoarthritis in $27(38 \%)$ patients, AVN in $6(8 \%)$ cases, and Legg-Perthes-Calve disease in $6(8 \%)$ cases. In the SPS ${ }^{\circledast}$ group, the most frequent etiologies were primary osteoarthritis in $456(80 \%)$ patients, DDH in 18 (3.5\%) patients, AVN in $65(13 \%)$ cases, and Legg-Perthes-Calve disease in 6 cases $(1 \%)$. Patients in the custom group were significantly younger $(p<0.001)$ and more frequently suffered DDH $(p<0.001)$. All patients had an HA-coated acetabular component (APRIL ${ }^{\circledR}$, Symbios, Switzerland) with a Biolox delta ceramic head and liner (CeramTec, Germany). A $28 \mathrm{~mm}$ head was used for cup diameters under $44 \mathrm{~mm}$, a $32 \mathrm{~mm}$ head for cup diameters under $50 \mathrm{~mm}$, and a $36 \mathrm{~mm}$ for larger cups. All the surgical procedures were performed by one surgeon (E. Sariali) who used a minimally invasive direct anterior approach (DAA), with patients positioning supine on a traction table [11]. Prior to surgery, patients had a low-dose CT scan [12] and $3 \mathrm{D}$ planning using the HIP-PLAN ${ }^{\circledR}$ software [13] to determine the prosthetic components size and position and to anticipate any surgical difficulties. The study was conducted according to the
French bioethics law (Article L. 1121-1 of law no 2004-806, August 9, 2004), and an approbation was accorded by the patient protection committee responsible for this hospital.

Surgical planning. Cup implantation was simulated. The 3D-cup template was positioned relative to the medial acetabular wall, which was not breached. The cup was completely covered by the acetabular bone in order to avoid any impingement with surrounding soft tissues, especially the psoas tendon. The goal was to restore the native acetabular anteversion and to achieve a cup inclination of $40^{\circ}$ (Fig. 6.1). In patients with developmental dysplasia of the hip (DDH), a standard $20^{\circ}$ acetabular anteversion was planned. The stem size was chosen to maximize both the fit and fill in the metaphysis. To determine the cranio-caudal stem positioning, a colored image mode reflecting the density of the bone (based on Hounsfield units) in contact with the stem was used. To achieve good primary mechanical stability, the surgeon assumed that the stem should be in contact with highly dense (i.e., cortical) bone at least on the stem's lateral flare and the calcar (Fig. 6.2). The goal was also to restore the global hip offset corresponding to the sum of the acetabular offset and the femoral offset. Indeed, if a medial translation of the cup was required in order to achieve a good bony coverage of the cup, the femoral offset was increased by the same amount in order to restore the native global offset. Once the cup and the stem implantation were simulated, four points were determined during the preoperative planning in order to simulate the alteration of the hip anatomy induced by the arthroplasty (Fig. 6.3): (1) the centers of the acetabulum (Ac) and the cup (Cc) - the vector between these two points $\mathrm{Ac}$ and $\mathrm{Cc}$ was labeled acetabular displacement (AD); (2) the centers of the femoral head (FHc) and the femoral ball (FBc) - the vector between these two points FHc and $\mathrm{FBc}$ was labeled femoral head displacement (FHD). The global femoral displacement (FD) was measured as the sum of these two vectors AD and FHD. The goal was to achieve FD = 0, which means that the relative positions of the two native centers Ac and FHc were not altered by THA. A rotational analysis of the entire lower limb was 


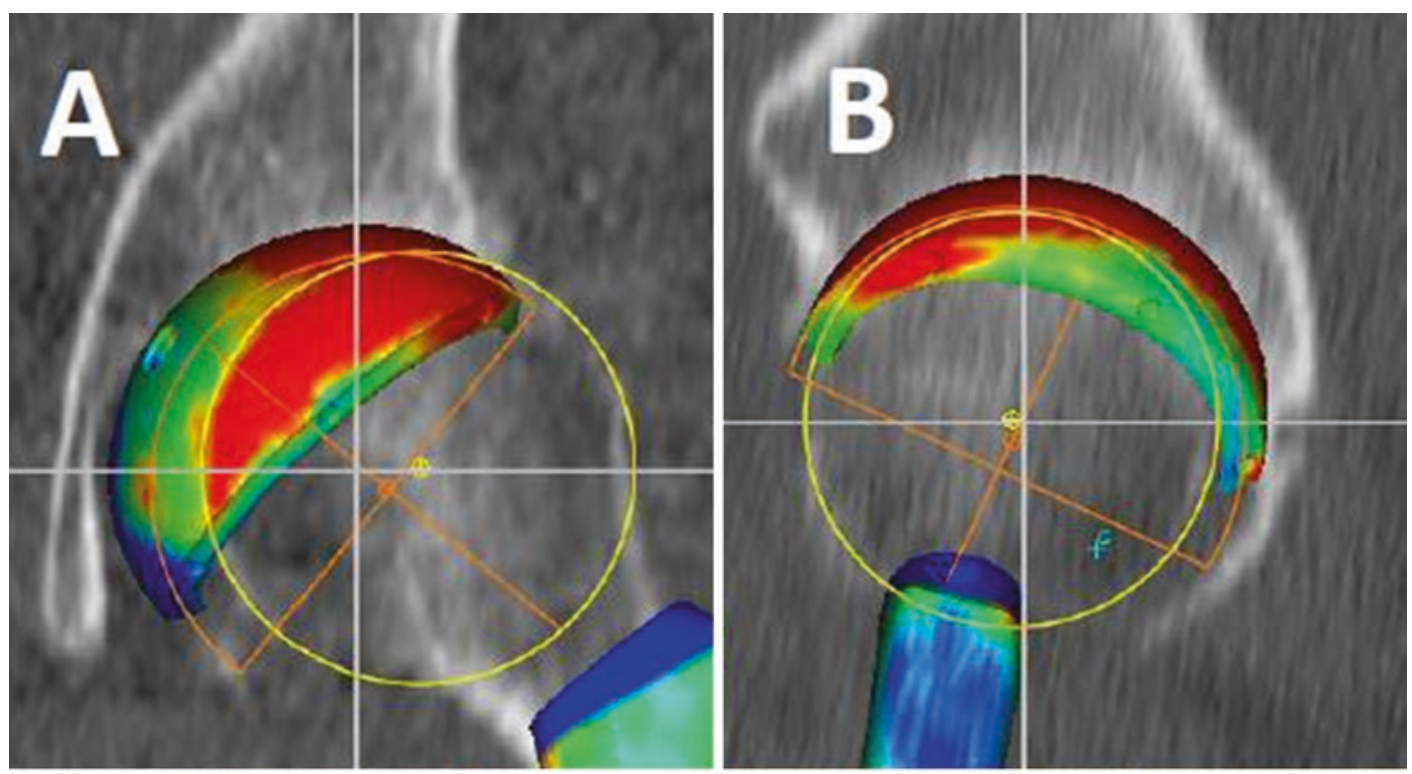

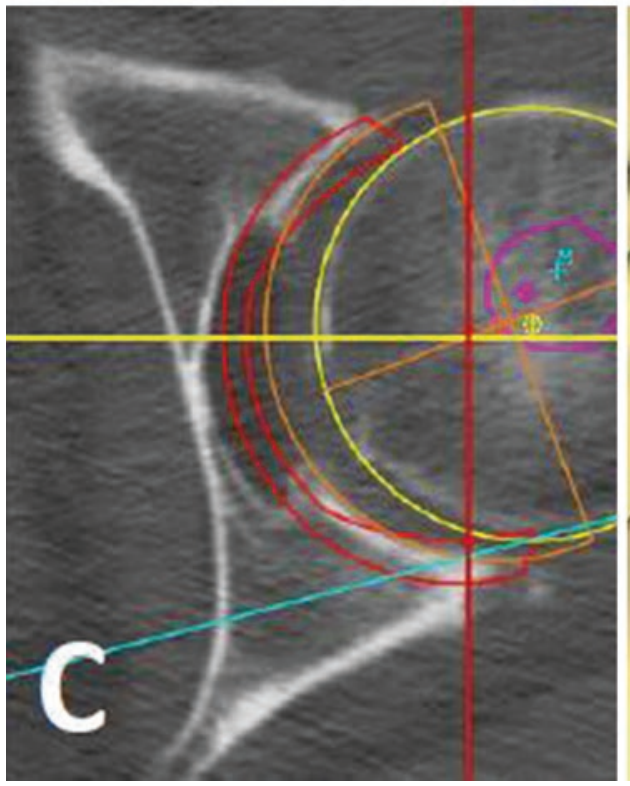

Fig. 6.1 Simulated 3D cup position (Coronal view (a), sagittal view (b), axial view (c) and 3D view (d)). To achieve primary stability, we assumed that the cup had to be in contact with highly dense bone on at least three points: the two

also performed, which included measuring the acetabular anteversion, the femoral neck anteversion, and the foot orientation angle-defined as the angle between the bi-malleolar axis and the posterior knee bicondylar plane line (Fig. 6.4). Based on previously reported results regarding the dislocation risk of DAA-THA [14], the goal walls and the roof. The 3D position of the cup was determined by measuring the distance from the edge of the cup to the edge of the bony acetabulum, especially relatively to the two walls (black arrows) and the lateral part of the roof

was to restore the native femoral anteversion unless the femoral displacement (FD) in the anteroposterior direction was above $8 \mathrm{~mm}$. This situation is typically observed when a posterior shift in the hip's center of rotation (COR) is combined with an increase in the femoral anteversion. In this case, a custom stem with a retroverted 


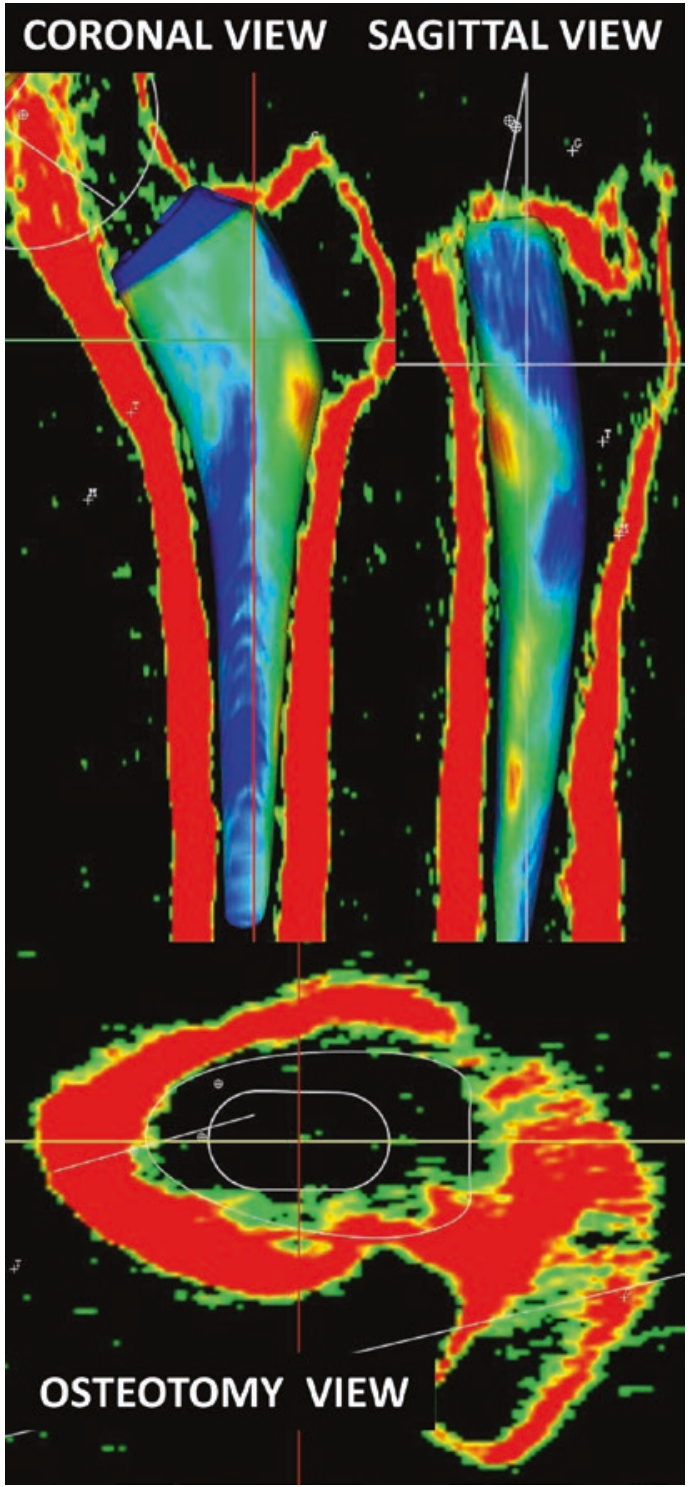

Fig. 6.2 3D planning of the stem including a coronal and sagittal analysis. A view of the osteotomy plane was available at the time of surgery to assist in controlling stem torsion

neck was used to make the femoral ball center coincide with the cup center (Fig. 6.5). When a decreased foot angle was observed, the femoral anteversion was slightly decreased in order to achieve a $15^{\circ}$ foot orientation. For the femoral ball, four lengths could be used to alter neck length: $-4 \mathrm{~mm}, 0,+4 \mathrm{~mm}$, and $+8 \mathrm{~mm}$. A custom femoral stem was used if the $3 \mathrm{D}$ reconstruction was not achievable with our standard stem $\left(\mathrm{SPS}^{\circledR}\right.$, Symbios SA). In this purpose, we used a tolerance of $15 \%$ for the offset and length and a tolerance of $6 \mathrm{~mm}$ for the anteroposterior position of the hip rotation center. The stem was designed to maximize the fit and fill in the metaphyseal zone (20 $\mathrm{mm}$ on each side of the middle of the lesser trochanter). The minimum stem length was calculated to withstand the fatigue tests.

Surgical technique. Minimally invasive DAA was used for all the patients. The cup was leveled with the tear drop and placed relative to the medial acetabular wall. The surgeon visually reproduced the preoperative planned position of the cup relatively to the acetabular rim by checking the distances from the edge of the cup to the acetabular roof and to the anterior and the posterior walls, using a 3D view of the simulated cup as a guide. The surgeon checked the final stem position with two parameters measured during the 3D planning. Firstly, he measured the distance from the top of the lesser trochanter to the top of the stem. Secondly, in order to control the stem anteversion, the surgeon performed a visual check of the position of the stem relative to the femur cross section corresponding to the neck osteotomy; this view was planned preoperatively. For the custom stems, only one custom rasp was used for the femur preparation. The postoperative protocol included full immediate weight-bearing for all patients.

Quality control of the implantation. In order to assess the accuracy of anatomically reconstructing the hip when using custom stem, we compared the native and prosthetic anatomical parameters in 30 consecutive patients who underwent a custom THA. For this, a pre- and postoperative CT scans were matched with the HIP-PLAN ${ }^{\circledR}$ software by independently aligning pelvic and then femoral bony landmarks (Fig. 6.6). We measured limb length discrepancy and changes to femoral offset and femoral anteversion.

Anticipation of surgical difficulties. The surgeon tried to forecast the following difficulties: (1) femoral perforation or femoral fracture which, in our experience, are more likely to occur if three conditions are combined: (a) a 


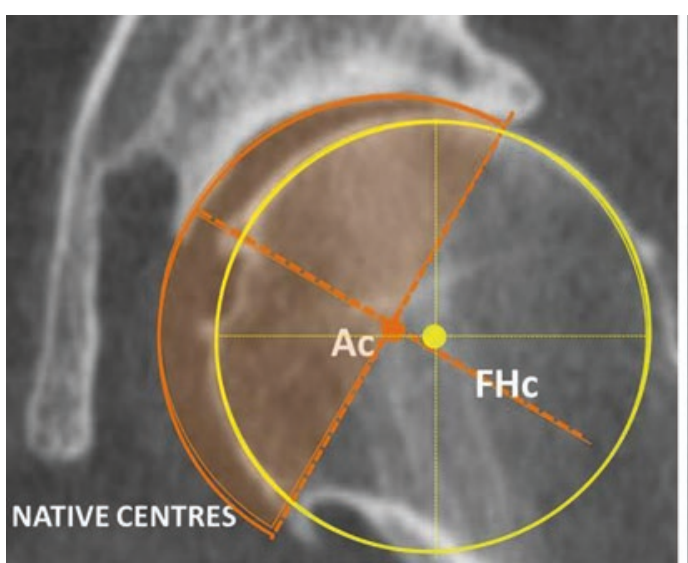

Fig. 6.3 The centers of the native acetabular (Ac) and femoral head $(\mathrm{FHc})$ were determined. The distance between these two points was labeled initial displacement (ID) which corresponds to the articular surfaces wear. The centers of the final cup $(\mathrm{Cc})$ and femoral prosthetic ball $(\mathrm{FBc})$ were determined. The vector $\mathrm{AcCc}$ was labeled

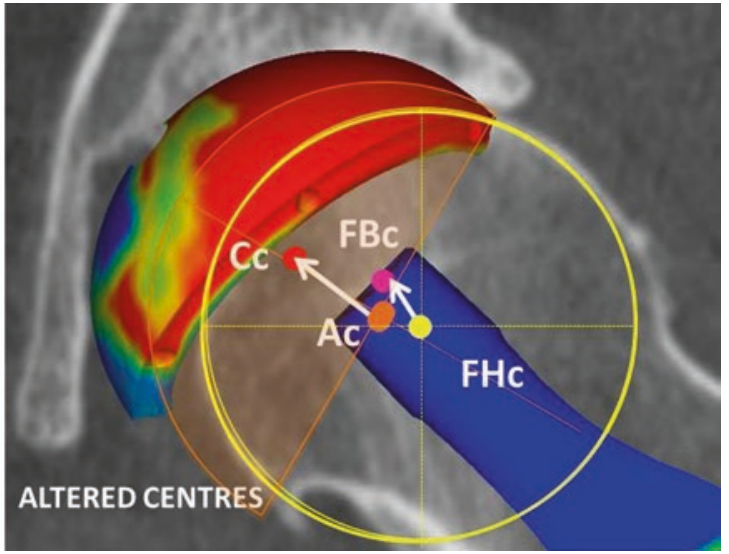

acetabular displacement (AD). The vector $\mathrm{FHcFBc}$ was labeled femoral head displacement (FHD). The global femoral displacement FD was measured as the sum of AD and FHD. We aimed for FD =0. A XL head (long neck) is simulated
Fig. 6.4 Lower limb torsion was analyzed including the acetabular anteversion, the femoral anteversion, and the foot orientation

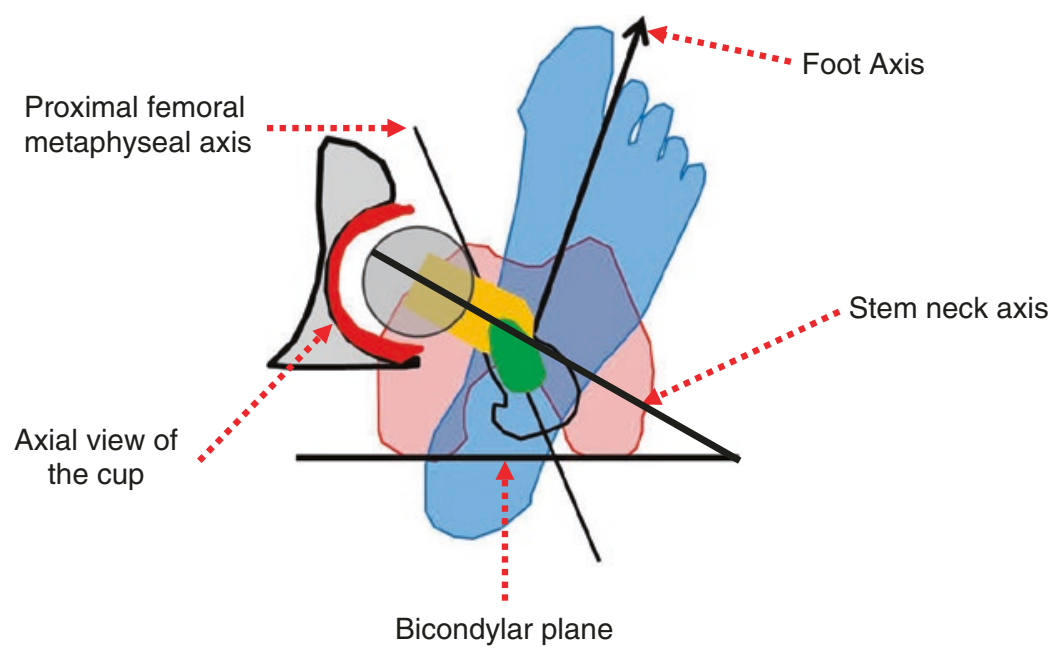

high anterior curvature of the femur, (b) a high density of cancellous bone at the upper part of the femur, and (c) and a narrow femoral isthmus. In these cases, before starting the rasping procedure, the femoral canal was reamed using a power tool and flexible reamers. (2) Any difficulties in simultaneously restoring femoral offset and length, especially in patients who have a femoral canal size incongruent with femoral offset (i.e., large femoral canal and low offset and vice versa). (3) Inappropriate final femoral anteversion $\left( \pm 10^{\circ}\right.$ compared to the native femoral anteversion) as a result of abnormal femoral torsion. For these cases, a suitably retroverted or anteverted neck was proposed to reduce the risk of prosthetic impingement and therefore increase stability.

Clinical assessment. Patients were assessed at the last follow-up with two self-completed questionnaires: the Harris Hip Score ( 0 worst and 100 best) and the Oxford Hip Score (0 worst and 60 best). 


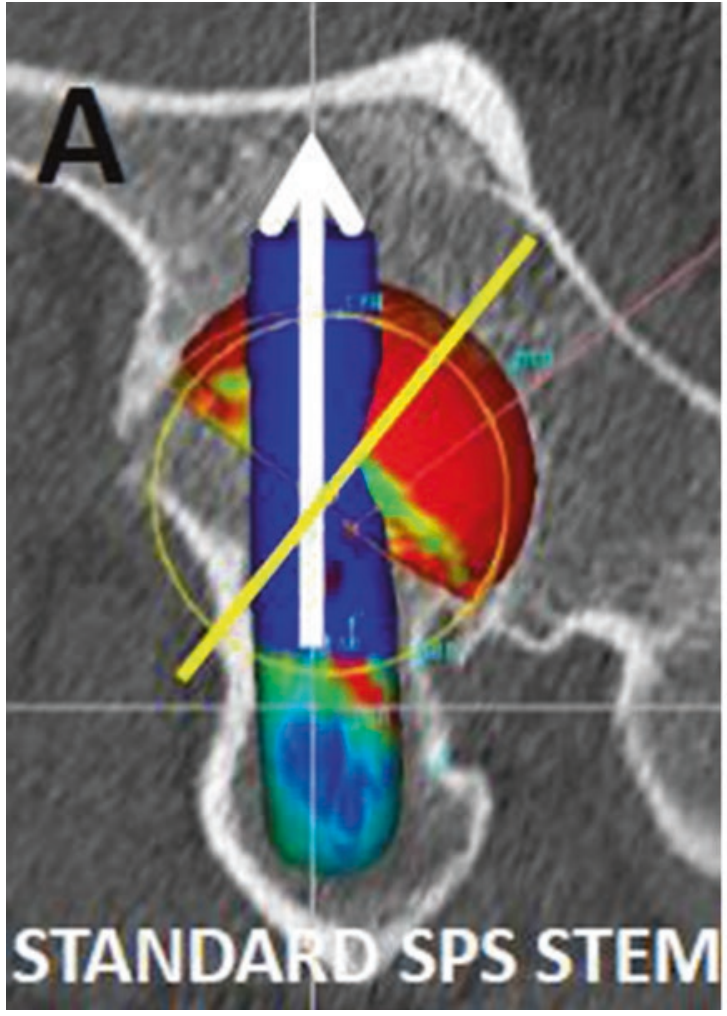

Fig. 6.5 This case shows a dysplastic hip with a mismatch between the femoral ball center and the cup center due to excessive antetorsion of the proximal metaphyseal part of the femur. The acetabular reaming procedure generates a posterior translation of the center of rotation and

Statistical analysis method. Pearson correlation coefficient was used to study the relationship between two variables (preoperative and postoperative anteversion values). Surgical precision was defined by assessing the difference in matched anatomical parameters between the planned and the postoperative values (mean \pm SD). Data were assessed for normality using the Ryan-Joiner and Shapiro-Wilk tests. For normally distributed variables, when two groups had the same variances, differences between them were analyzed using Student's $t$-test. For abnormally distributed variables or normally distributed variables with different variances, the Mann and Whitney test was used. A

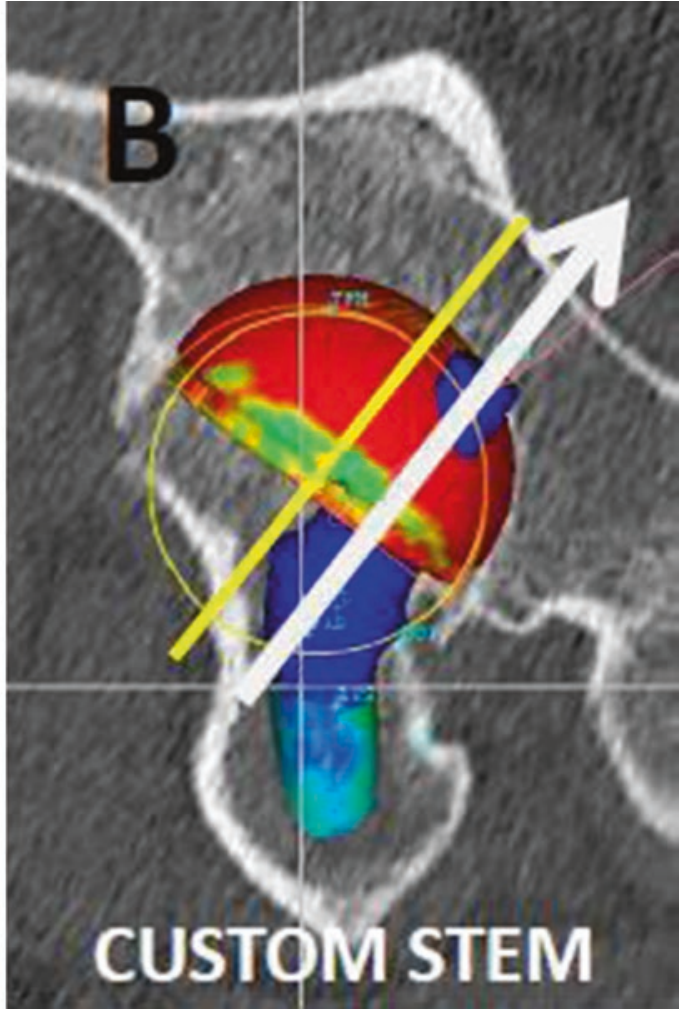

consequently an anterior hip instability. A custom stem with a retroverted neck (b) was used to make the femoral ball center coincide with the cup center at contrary to a standard straight-neck stem (a)

$p$-value of less than 0.05 was considered significant. Statistical analysis was performed with JMP software (version-11; SAS Institute).

\subsection{Results}

Implantation accuracy. There was excellent agreement between the planned and the performed femoral stem anteversions with an implantation accuracy of $1^{\circ}\left( \pm 4^{\circ}\right)$. The difference between the planned $\left(20^{\circ} \pm 8^{\circ}\right)$ and the postoperatively measured femoral anteversion $\left(21^{\circ} \pm 8^{\circ}\right)$ was not statistically significant $(p=0.3)$, and their correlation was very strong 

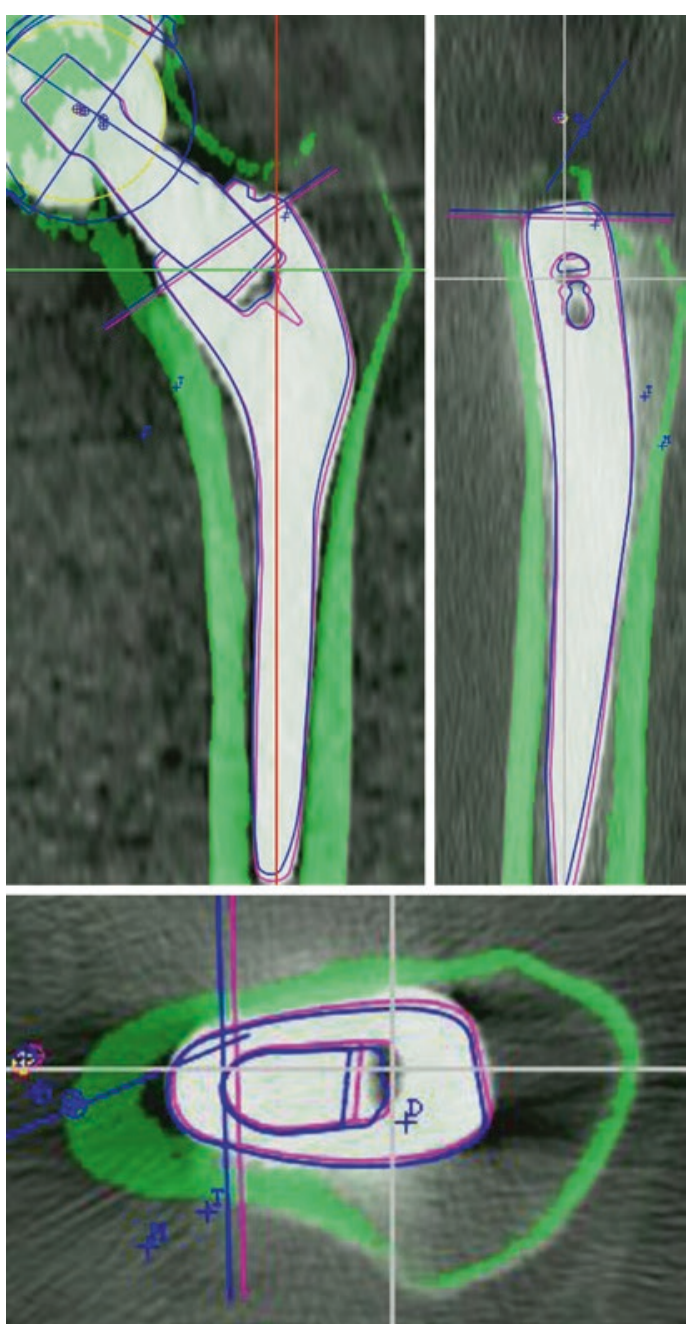

Fig. 6.6 Matching of preoperative and postoperative CT scans was performed with the HIP-PLAN ${ }^{\circledR}$ software in a group of 30 patients in order to compare the planned and performed positioning of components

$(r=0.9, p<0.001)$ (Fig. 6.7a). There was excellent agreement between the planned and performed lower limb length (LL) with an implantation accuracy of $-0.6 \pm 2.5 \mathrm{~mm}$. There was no significant difference between the planned $(5 \pm 4.6 \mathrm{~mm})$ and the executed $(4.4 \pm 5.5 \mathrm{~mm}) \mathrm{LL}$ $(p=0.3)$, and the correlation between them was found very strong $(r=0.9, p<0.001)$ (Fig. 6.7b). Last, there was excellent agreement between the planned and performed femoral offset with an implantation accuracy of $-1.2 \pm 2.4 \mathrm{~mm}$. There was no significant difference between the planned FO value $(43.3 \pm 6.8 \mathrm{~mm})$ and the postoperative one $(42.1 \pm 7.0 \mathrm{~mm})(p=0.3)$, and furthermore, a very strong correlation between these two values was found ( $r=0.95, p<0.001)$ (Fig. 6.7c).

Anticipation of surgical difficulties. The main anatomic reasons that led to use a custom stem were: (1) torsional abnormalities of the proximal femur that prevented restoring a planned femoral anteversion (Fig. 6.8) and potentially made the patient prone to dislocation or foot malorientation, (2) severe coxa vara or coxa valga making the simultaneous restoration of femoral offset and length challenging when using conventional stems (Fig. 6.9), and (3) severe outlier morphotypes such as dwarf and giant patients where off-the-shelf stems are inappropriate and either too big or too small, respectively.

Clinical outcomes. At 5 years \pm 2 mean follow-up, no stem was revised for an aseptic reason, no dislocation occurred, no patient complained of limb length discrepancies, and excellent clinical results were achieved. The mean HHS improved from 30 to $93( \pm 16)$ and the Oxford score improved from 23 to $56( \pm 9)$.

\subsection{Discussion}

The main results from our study were that (1) $12 \%$ of patients required a custom stem to reconstruct their native femoral anatomy, the main reasons being torsional abnormalities and severe coxa vara or coxa valga; (2) the manual implantation (technology free) of custom stem was precise; and (3) performing anatomic restoration of hip biomechanics using 3D planning, intraoperative checks, and custom implants resulted in excellent functional outcome for patients with atypical proximal femoral anatomy.

The main limitation of this study is that our results are implant and patient specific. Our 


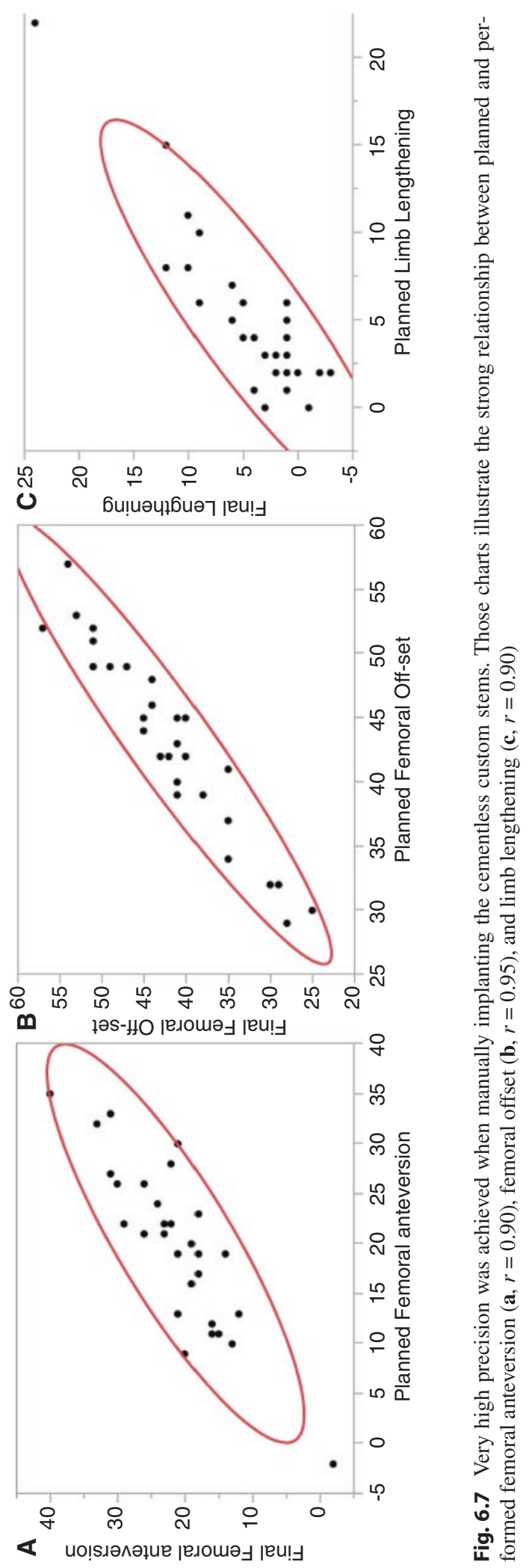




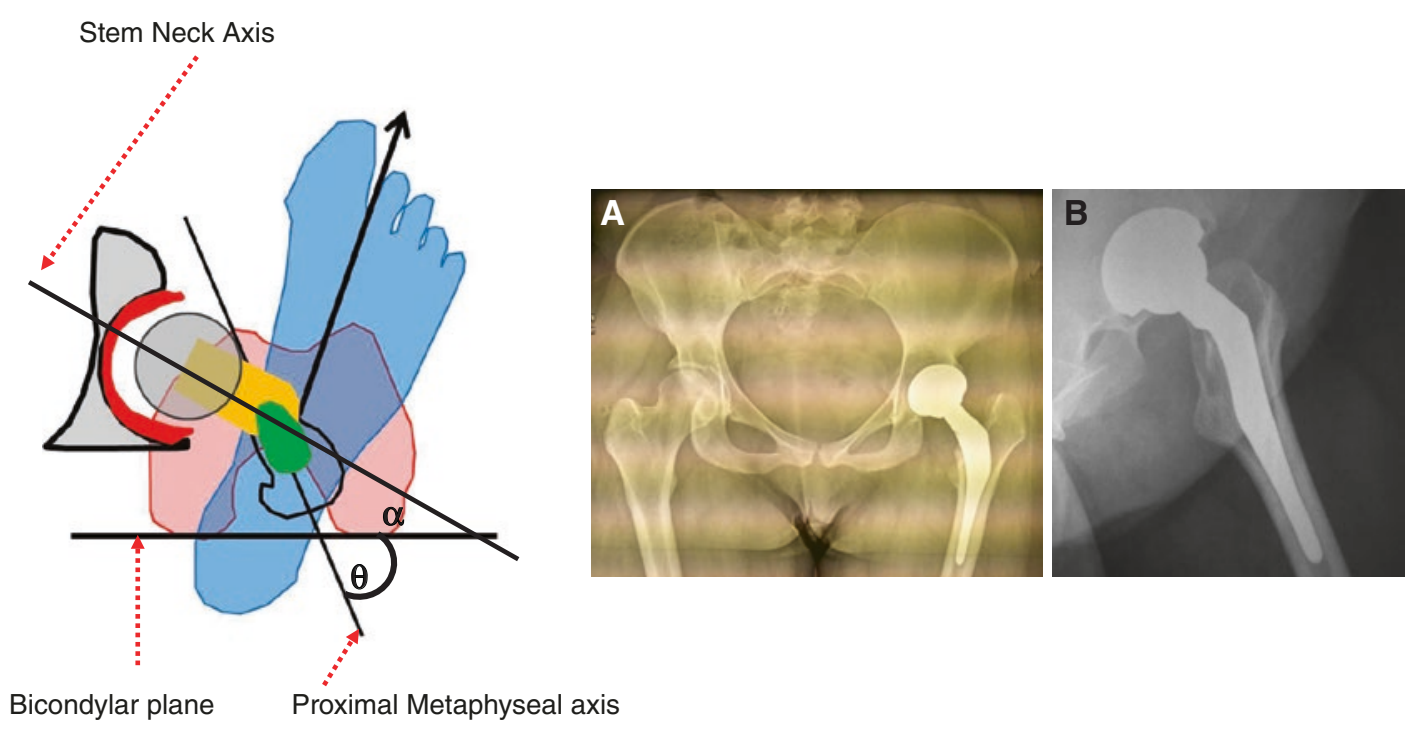

Fig. 6.8 The post operative radiographies are presented: (a) Antero-posterior view (b) lateral view. Illustration of a 3D plan for a patient with a severe torsional disorder with a proximal femoral metaphyseal version of $63^{\circ}-$ increased by $40^{\circ}$ compared to native femoral antever-

results regarding the proportion of patients needing a custom stem, and the reasons for this, only apply to the SPS stem design. Different results would probably be found with other stem designs.

The accuracy for anatomically reconstructing the hip with custom stem technology was judged to be excellent. This accuracy compares well with previously reported results for 3D planningbased off-the-shelf THA [4, 11]. However, patients in the custom group had complex hip anatomy, primarily regarding their proximal femoral morphology, and restoration of normal biomechanics would not be achievable with conventional implant designs such as the SPS ${ }^{\circledR}$ stem (anatomic design).

Few studies have assessed the accuracy of postoperative hip anatomical restoration using a CT scan, as it requires careful 3D analysis of preoperative anatomy and accurate matching. Contrary to literature on optimal acetabular cup positioning, there is no "safe zone" recommended for femoral anteversion. In this study, we propose a new method for defining the target femoral anteversion. Surgeons should compensate for changes in the acetabular center sion. A $40^{\circ}$ retroversion of the neck relatively to the shaft was required to stabilize the hip. The use of standard straight-neck SPS Stem would have led to a $40^{\circ}$ excess in stem anteversion. A $40^{\circ}$ retroversion of the neck relatively to the shaft was required to stabilize the hip

induced by reaming. Typically, acetabular preparation induces a posterior, medial, and cranial shift of the hip center of rotation. In response, we advise that femoral offset and anteversion/ retroversion are adapted accordingly during 3D planning. In the case of a high-grade dysplastic hip (dislocated), a $15-20^{\circ}$ anteversion was aimed for.

Kirshnan et al. [15] reported that the intracanalar (femur flares and volume) and the extracanalar proximal femur anatomy (femoral offset, neck length, and femoral anteversion) are not correlated, suggesting that the same proximal femur volume may correspond to a highly variable femoral offset. Interestingly, Sariali et al. [13] showed that for a given stem size, the required range for stem $\mathrm{FO}$ was $22 \mathrm{~mm}$ in order to restore accurately the patient native FO. Hence, for outlier patients, custom stems are the favored solution. They allow the surgeon to accurately address the extramedullary anatomy independent of the intracanalar anatomy, while avoiding the complications related to the use of modular necks such as modular neck fractures and adverse local tissue reaction to metal debris. 

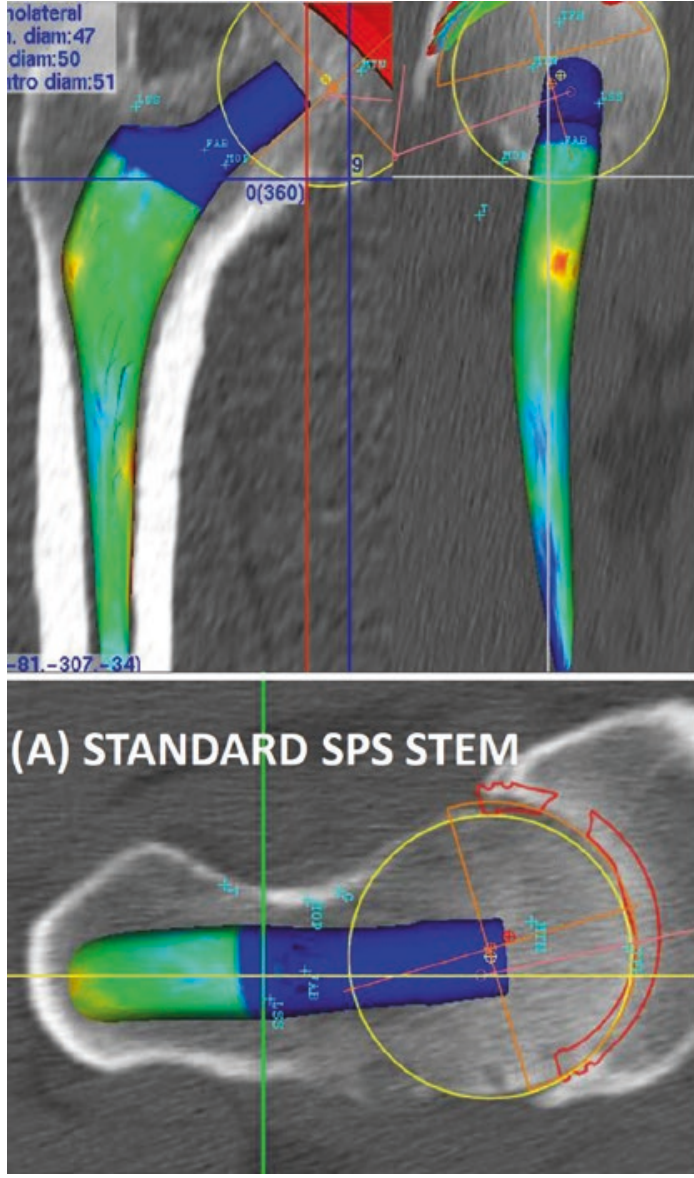

Fig. 6.9 The 3D plan in this case shows a hip with severe coxa vara and a high femoral offset. The standard $129^{\circ}$ CCD angle stem (a) would not allow the restoration of

\subsection{Conclusion}

Custom stem technology is a reliable solution to treat degenerated hip patients having an atypical hip anatomy. Approximately, $12 \%$ of patients require a custom stem to achieve an accurate reconstruction of their proximal femur anatomy. Performing 3D planning for all osteoarthritic hip patients and checking intraoperative anatomical parameters are key steps to anticipate the surgical difficulties, select the appropriate implants, and restore normal hip biomechanics.
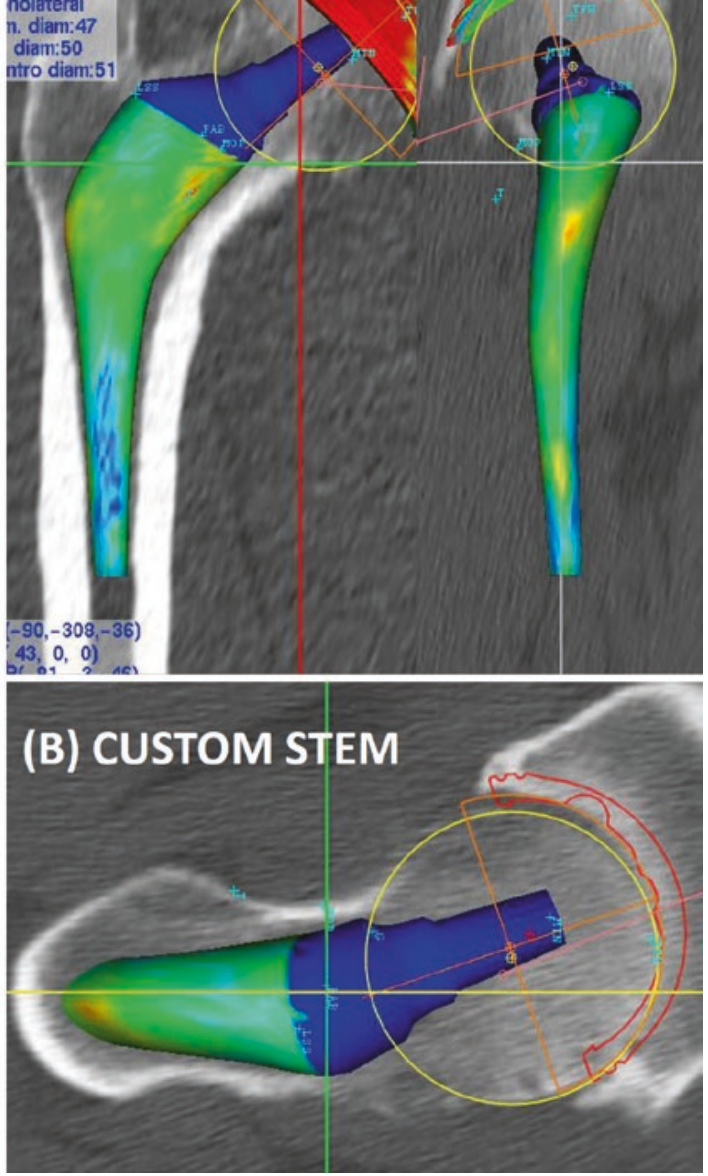

proximal femur anatomy. The custom stem (b) included a higher offset and a $15^{\circ}$ retroversion of the neck relative to the shaft

\section{References}

1. Argenson J, Ryembault E, Flecher X, Brassart N, Parratte S, Aubaniac J. Three-dimensional anatomy of the hip in osteoarthritis after developmental dysplasia. J Bone Joint Surg Br. 2005;87(9):1192-6.

2. Husmann O, Rubin P, Leyvraz P, de Roguin B, Argenson J. Three-dimensional morphology of the proximal femur. J Arthroplast. 1997;12:444-50.

3. Schmidutz F, Graf T, Mazoochian F, Fottner A, BauerMelnyk A, Jansson V. Migration analysis of a metaphyseal anchored short-stem hip prosthesis. Acta Orthop. 2012;83(4):360-5.

4. Sariali E, Mouttet A, Pasquier G, Durante E. Three dimensionnal hip anatomy in osteoarthritis. Analysis of the femoral off-set. J Arthroplast. 2009;24(6):990-7. 
5. Asayama I, Chamnongkich S, Simpson K, Kinsey T, Mahoney O. Reconstructed hip joint position and abductor muscle strength after total hip arthroplasty. J Arthroplast. 2005;20:414-20.

6. Sariali E, Klouche S, Mamoudy P. Ceramic-onceramic total hip arthroplasty: is squeaking related to an inaccurate three-dimensional hip anatomy reconstruction? Orthop Traumatol Surg Res. 2012;100(4):437-40.

7. Sariali E, Klouche S, Mouttet A, Pascal-Moussellard H. The effect of femoral offset modification on gait after total hip arthroplasty. Acta Orthop. 2014;85(2):123-7. Epub 2014/02/26.

8. Kwon YM, Khormaee S, Liow MH, Tsai TY, Freiberg AA, Rubash HE. Asymptomatic pseudotumors in patients with taper corrosion of a dual-taper modular femoral stem: MARS-MRI and metal ion study. J Bone Joint Surg Am. 2016;98(20):1735-40. Epub 2016/11/22.

9. Flecher X, Pearce O, Parratte S, Aubaniac J, Argenson J. Custom cementless stem improves hip function in young patients at 15-year followup. Clin Orthop Relat Res. 2010;468(3):747-55.

10. Dessyn E, Flecher X, Parratte S, Ollivier M, Argenson JN. A 20-year follow-up evaluation of total hip arthroplasty in patients younger than 50 using a custom cementless stem. Hip Int. 2018;23:1120700018803290. Epub 2018/10/24.

11. Sariali E, Catonne Y, Pascal-Moussellard H. Threedimensional planning-guided total hip arthroplasty through a minimally invasive direct anterior approach. Clinical outcomes at five years' follow-up. Int Orthop. 2017;41(4):699-705. Epub 2016/06/18.

12. Huppertz A, Lembcke A, Sariali E, Durmus T, Schwenke C, Hamm B, et al. Low dose computed tomography for 3D planning of total hip arthroplasty: evaluation of radiation exposure and image quality. $\mathrm{J}$ Comput Assist Tomogr. 2015;39(5):649-56.

13. Sariali E, Mouttet A, Pasquier G, Durante E, Catonne Y. Accuracy of reconstruction of the hip using computerised three-dimensional pre-operative planning and a cementless modular-neck stem. J Bone Joint Surg Br. 2009;91(3):333-40.

14. Sariali E, Klouche S, Mamoudy P. Investigation into three dimensional hip anatomy in anterior dislocation after THA. Influence of the position of the hip rotation centre. Clin Biomech. 2012;27(6):562-7.

15. Krishnan S, Carrington R, Mohiyaddin S, Garlick N. Common misconceptions of normal hip joint relations on pelvic radiographs. J Arthroplast. 2006;21:409-12.

Open Access This chapter is licensed under the terms of the Creative Commons Attribution 4.0 International License (http://creativecommons.org/licenses/by/4.0/), which permits use, sharing, adaptation, distribution and reproduction in any medium or format, as long as you give appropriate credit to the original author(s) and the source, provide a link to the Creative Commons license and indicate if changes were made.

The images or other third party material in this chapter are included in the chapter's Creative Commons license, unless indicated otherwise in a credit line to the material. If material is not included in the chapter's Creative Commons license and your intended use is not permitted by statutory regulation or exceeds the permitted use, you will need to obtain permission directly from the copyright holder.

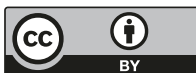

